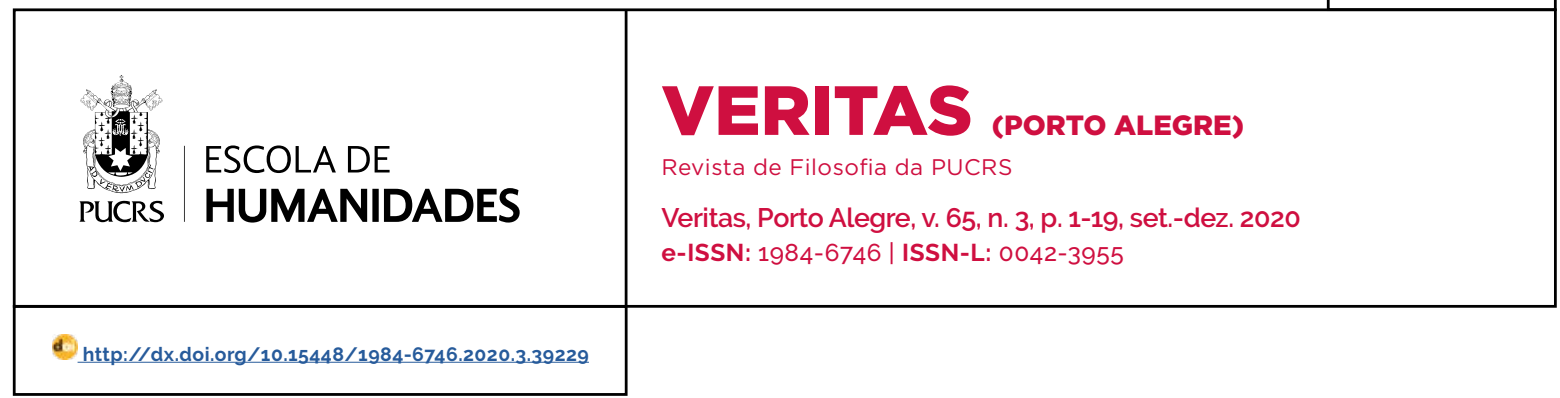

\title{
L'Approche de la « distance » dans les écrits littéraires de Michel Foucault, ou comment inscrire l'événement dans la structure $^{1}$
}

\author{
A abordagem da « distância » nos escritos literários de Michel Foucault, ou como \\ inscrever o acontecimento na estrutura
}

The « distance » approach in Michel Foucault's literary writings, or how to inscribe the event in the structure

\section{Gabriela M. Jaquet ${ }^{2}$ orcid.org/0000-0002-5066-875X gabrielajaquet@hotmail.com}

\author{
Norman R. Madarasz ${ }^{3}$ \\ orcid.org/0000-0002-7574-3744 \\ norman.madarasz@pucrs.br
}

Reçu en: le 26 Sept. 2020 Approuvé en: le 31 Oct. 2020 Publié dans: le 12 Jan. 2021
Résumé: Depuis la publication des Dits et écrits, la place de la littérature dans l'œuvre de Michel Foucault a une position bien assignée dans les recherches académiques, où l'on souligne surtout l'approche nietzschéenne de la lecture qu'a proposé le philosophe. Prenant appui sur des nouvelles publications et sur des manuscrits inédits, notre texte envisage ce thème sous un autre prisme, à partir du structuralisme de l'analyse littéraire et de l'enjeu philosophique de la revue Tel Quel dans son rapport avec le Nouveau Roman. En repérant l'importance du concept de « distance » dans ses écrits sur la littérature, nous discuterons comment plusieurs des notions créées par Foucault se trouvent en dialogue avec cette constellation théorique plus large. Nous y établirons ainsi le rapport entre la structure et l'événement, la paire conceptuelle au centre des débats philosophiques des années 1960, qui nous guidera dans la compréhension des tensions et des adhésions de la méthodologie foucaldienne à l'égard de l'analyse structurale.

Mots-clés: Analyse littéraire. Structuralisme. Distance. Événement. Tel Quel. BnF Inédits.

Resumo: Desde a publicação dos Ditos e Escritos, o lugar da literatura na obra de Michel Foucault possui uma posição bem definida nas pesquisas acadêmicas, nas quais se ressalta sobretudo a abordagem nietzscheana da leitura proposta pelo filósofo. Recorrendo a novas publicações e aos manuscritos inéditos do Fonds Foucault-BnF, este texto enfocará o tema da literatura sob um outro prisma, à partir do estruturalismo da análise literária e do contexto filosófico da revista Tel Quel em sua relação com o Nouveau Roman. Enfatizando a importância do conceito de "distância" em seus escritos sobre a literatura, discutir-se-á como várias das noções criadas por Foucault se encontram em diálogo com esta constelação teórica mais ampla. A relação entre a estrutura e o acontecimento, enquanto par conceitual que se encontrava no centro dos debates filosóficos nos anos 1960, nos guiará na compreensão das tensões e das adesões da metodologia foucaltiana no que tange à análise estrutural. Palavras-chave: Análise literária. Estruturalismo. Distância. Acontecimento. Tel Quel; BnF Inéditos.

Abstract: Since the publication of the Dits et écrits, the place held by literature in Michel Foucault's work has been clearly recognized by academic research, which has emphasized the Nietzschean approach of the reading made by the philosopher. Based on new publications and unpublished manuscripts, our paper considers this theme from another angle, that is, from structuralism in literary analysis and the philosophical stakes of the periodical Tel Quel in its relation with the French Nouveau Roman. By highlighting the importance of the concept of "distance" in his writings on literature, we aim to discuss how several of the notions created by Foucault are seen to be in dialogue with this larger theoretical constellation. As a case in point, we show the relation between structure and event, the conceptual 
pair at the center of philosophical debates in the 1960s, which guides us in the understanding of the tensions and adhesions of Foucault's methodology with regard to structural analysis.

Keywords: Literary analysis. Structuralism. Distance. Event. Tel Quel. BnF Fonds Michel Foucault.

\section{Avant-propos}

Depuis la publication des Dits et écrits, la place de la littérature dans l'œuvre foucaldienne a une position bien assignée parmi les recherches académiques, où l'on analyse surtout les travaux du philosophe concernant G. Bataille, M. Blanchot, A. Artaud, G. Nerval, P. Klossowski, S. Mallarmé, ou le M. de Sade pour les connecter à l'approche nietzschéenne de la lecture qu'a proposé le philosophe. Néanmoins, avec la publication de La Grande étrangère : à propos de littérature, en 2013. de Folie, langage, littérature, en $2019^{4}$, ou avec l'accès aux manuscrits déposés à la Bibliothèque nationale de France, on peut constater également que la recherche foucaldienne autour de la littérature ${ }^{5}$ prend ses racines non pas seulement dans les œuvres en quelque sorte premières de ces écrivains, mais dans le débat théorique ouvert par le structuralisme de la Nouvelle Critique, et par les écrivains liés à la revue Tel Quel et au formalisme littéraire entre 1950 et 1965.

En repérant la récurrence et l'importance du concept de « distance » dans les écrits de Foucault sur la littérature et à partir de la mise en relief du caractère structural de sa philosophie, le propos de notre article sera de mettre en valeur certains de ses concepts distribués dans un ensemble de textes autour de la revue Tel Quel. Parmi ceux-ci, nous rejoindrons spécialement celui intitulé « Distance, aspect, origine », paru en 1963. Nous discuterons ainsi, dans un premier temps, des éléments les plus généraux concernant l'analyse littéraire en France afin de comprendre le contexte de la critique que Foucault adressera à l'écrivain Alain Robbe-Grillet, luimême rattaché au mouvement avant-gardiste du Nouveau Roman. Dans un deuxième temps, plus spécifiquement à propos de l'article de 1963, nous analyserons quelques concepts de la nouvelle méthodologie que présente le philosophe, basée alors sur la notion de « distance » : le concept de « réseau », l'enjeu de la « lacune » et la question de « l'aspect ». À travers cette approche nous suivrons parallèlement la configuration générale du rapport entre la structure et l'événement. Cette paire conceptuelle, au centre des querelles philosophiques dans les années 1960, nous guidera dans la compréhension des tensions et des adhésions de la méthode foucaldienne à l'égard de l'analyse structurale.

« L>écart, la distance, l>intermédiaire, la dispersion, la fracture, la différence ne sont pas les thèmes de la littérature $d>a u j o u r d>$ hui ; mais ce en quoi le langage maintenant nous est donné et vient jusqu>à nous : ce qui fait qu>il parle $\gg^{6}$. Dans une grande partie de ses écrits et de ses notes manuscrites du début des années 1960 , Foucault fait usage du concept de « distance » en référence à « l'espace du langage » littéraire. Tel concept prendra plusieurs noms - vide, béance, manque, écart, lacune, intermédiaire, creux - jusqu'à configurer, en 1964, la proposition d'une méthodologie issue de la sémiologie : la spatialisation de la distance ${ }^{7}$. La « distance » désigne, en général, l'espace non-représentationnel existant entre les mots et les choses : le propre du langage est de multiplier les chaînes du sens à partir d'un « creux » qui déstabilise les «accords » ordinaires de la signification, s'opposant ainsi au paradigme empiriste de l'identité qui concevait encore le langage comme une traduction transparente de la réalité. Il s'agit, chez Foucault, du mouvement

\footnotetext{
4 Michel Foucault, Folie, langage, littérature. Édition établie par H.-P. Fruchaud, D. Lorenzini et J. Revel, introduction de Judith Revel, Paris, Vrin, 2019 : M. Foucault, La Grande étrangère. À propos de littérature. Édition établie par Ph. Artières, M. Potte-Bonneville, J.-F. Bert et J. Revel, Paris, Éditions EHESS, 2013. La plupart des textes qui composent le premier recueil n'ont pas de datation exacte, situés entre 1960 et 1970 5 Sur le statut philosophique des analyses littéraires de Foucault, nous renvoyons à la recherche de Philippe Sabot : « La littérature aux confins du savoir. Sur quelques "dits et écrits" de Michel Foucault », in Pierre François Moreau (dir.), Lectures de Michel Foucault 3 : Sur les Dits et écrits, Lyon, ENS Éditions, 2003. pp. 17-33. ; « Notice » (Raymond Roussel), dans M. Foucault, OEuvres, vol. 1, Gallimard, « Bibliothèque de la Pléiade », Paris, 2015, pp. 1552-1662. Voir aussi : Judith Revel et Azucena G. Blanco, « Michel Foucault : dire politique, dire littéraire, dire philosophique », Revue internationale de philosophie, dossier « Michel Foucault : politique, littérature et philosophie », $\mathrm{n}^{\circ} 292,2 / 2020$.

6 M. Foucault, « Le langage de l'espace », Critique, n² 203, avril 1964, repris dans Dits et écrits, vol. 1, Paris, Gallimard, $1994,407-412$, ici p. 407.

7 M. Foucault, « Littérature et langage » (1964), in La Grande étrangère. Ȧ propos de littérature, op.cit., p. 120.
} 
de la construction progressive d'un domaine ontologique irréductible pour le langage. Cette configuration philosophique entraînant la notion de « distance » apparaît, avant la systématisation des Mots et les choses, dans Raymond Roussel, de 1963, où l'on repère l'usage structural du concept de «vide » en tant que mécanisme du langage (procédant par « prolifération » et « abolition » de la distance ${ }^{8}$, mais encore sous une certaine séparation entre ontologie et langage) ; puis dans les articles publiés à cette période, dont ceux sur Tel Quel et sur l'analyse littéraire sont un cas saillant par l'affirmation d'une « approche de la distance $\gg^{9}$ (servant à échapper aux reconductions « dialectiques » et « anthropologiques ») ; et, finalement, dans les conférences à Bruxelles en 1964 (qui radicalisent l'irréductibilité ontologique de « l'être du langage » par une intégration des « couches sémiologiques » à celles, foucaldiennes, des «formes de spatialisation $\left.\gg^{10}\right)$. $\dot{A}$ partir de 1966-1967, cet enjeu suivra le chemin de l'extralinguistique ${ }^{11}$, avec l'attention aux travaux de Jorge Luis Prieto et de John Austin, tout en déployant la transformation du problème du «langage » en celui du « discours $»^{12}$.

À propos du rapport entre Foucault et le structuralisme, nous pouvons aisément constater aujourd'hui qu'il a été très varié et ambigu ${ }^{13}$. Ici nous approcherons la méthode structurale en l'envisageant en tant que «question » toujours présente chez le philosophe et non comme vérité enfin apparue : comme l'affirme Étienne Balibar, la négation de l'étiquette fait partie du corps des hérésies du mouvement structuraliste lui-même ${ }^{14}$. En 1963, Roland Barthes qualifiait le structuralisme comme étant une activité ${ }^{15}$. En 1967. Gilles-Gaston Granger écrivait qu'il y avait des structuralismes ${ }^{16}$. Dans la même année, Foucault défendait le structuralisme par le néologisme de deixologie, celle-ci étant la « discipline générale du document en tant que document $\gg^{17}$ et « l'analyse de la structure documentaire $\gg$ des choses ${ }^{18}$. Le « document » est alors ce qui découpe et établit les objets étudiés (et non le contraire) : la « deixologie » n'interprète pas le document, elle doit constituer des groupes de relations ${ }^{19}$. Dans les notes manuscrites de sa conférence « Revenir à l'histoire $\gg^{20}$, prononcée au Japon en octobre 1970 (période où prolifèrent déjà ses critiques au structuralisme), le philosophe affirmera encore : « La comparaison structurale ne sert pas à établir une identité intemporelle, mais un système de différences liées, subordonnées, analysables. Il ne s'agit [pas] de substituer l'immobile au devenir : mais l'analyse des transformations à la constatation globale du changement $\gg^{21}$.

Dans le sillage des critiques classiques, comme celles de Paul Ricœur ou de Jean-Paul Sartre, les contestations du structuralisme attaquaient un fixisme ou un détérminisme trop généralisant crée

8 M. Foucault, Raymond Roussel, Paris, Gallimard, 1963, p. 23 et p. 47

9 M. Foucault, « Guetter le jour qui vient », La Nouvelle Revue française, n 130, octobre 1963, repris dans Dits et écrits, vol. 1, op.cit., ici p. 265 10 M. Foucault, « Littérature et langage », op.cit., p. 141.

11 Sur la problématique de l'extralinguistique, voir : Daniele Lorenzini, « Foucault et la force des mots : de l'extralinguistique à la subjectivation », Phantasia, « Michel Foucault et la force des mots », D. Lorenzini (dir.), Volume 8, 2019.

12 Voir : M. Foucault, « L'extralinguistique et la littérature » (pp. 223-242) et « L'Analyse littéraire et le structuralisme » (pp. 243-264), in Folie, langage, littérature, op.cit. Le domaine de l'extralinguistique est également discuté dans le texte inédit remontant au séjour du philosophe à Tunis : Le Discours philosophique, BnF, Fonds Foucault, NAF 28730, boîte LVIII, dossier 2. Cette boîte se compose aussi par le manuscrit du Cours de Tunis (dossier 1). Là, on trouvera quelques fois le mot « langage » biffé à la main et substitué par celui de « discours », ce qui corrobore l'idée qu'il y a une «transformation » des enjeux conceptuels au lieu d'un écartement de la problématique du langage à partir de la fin de années 1960

13 Malgré la distance prise par rapport au structuralisme notamment après 1970, on peut clairement voir que Foucault s'est bien reconnu dans la méthode structurale. Au-délà des deux recueils mentionnés, de 2013 et de 2019, nous pourrions citer aussi plusieurs autres exemples. Pour ceux plus « évidents », dans les Dits et écrits : « Entretien avec Madeleine Chapsal », de 1966 (vol. 1, pp. 513-518), « La philosophie structuraliste permet de diagnostiquer ce qu'est aujourd'hui », de 1967 (vol. 1, 580-584), «Interview avec Michel Foucault », de 1968 (vol. 1. pp. 651-662), « Linguistique et sciences sociales », de 1968 (vol. 1, pp. 821-842), « Revenir à l'histoire », de 1970 (vol. 2, pp. 268-281).

14 Étienne Balibar, « Le structuralisme: une destitution du sujet ? », Revue de métaphysique et de morale, n 45, 2005, pp. 5-22, p. 7.

15 Roland Barthes, «L'Activité structuraliste », Les lettres Nouvelles, n 32, 1963

16 Gilles-Gaston Granger, Pensée formelle et sciences de l'homme, Paris, Aubier-Montaigne, 1967, p. 2-6.

17 M. Foucault, «Structuralisme et analyse littéraire. Conférence prononcée au Club Tahar Haddad à Tunis le 4 février 1967 », in Folie, langage, littérature, op.cit., pp. 171-222, ici p. 175.

18 Ibid., p. 204. Nous soulignons.

19 Idem. Idée que dans L'Archéologie du savoir apparaît par un renversement : là, c'est le « monument » qui vient à la place du « document » (M. Foucault, L'Archéologie du savoir, Paris, Gallimard, 1969, p. 15, 182).

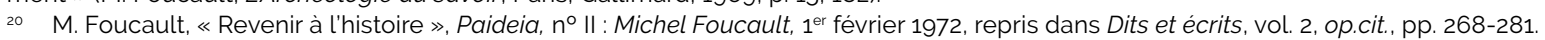

${ }_{21}$ M. Foucault, «Structuralisme et histoire », BnF, Fonds Foucault, NAF 28730, boîte LXX. 
par des grilles d'intelligibilité envisagées en tant que prisons où le réel frénétique devrait s'encadrer. L'important était néanmoins la création des catégories qui pouvaient alors ne plus supposer qu'elles seraient censées traduire une realité donnée d'avance : ni désignation extrinsèque ni signification intrinsèque ${ }^{22}$, comme l'a écrit Gilles Deleuze égalément en 1967. En remettant ainsi Foucault dans le contexte intellectuel français des années 1960, nous n'espérons pas l'apparition d'un fil explicatif causal des « influences », mais l'élucidation de la trame théorique qui entoure certains de ses choix épistémologiques. De la sorte, et tel que l'affirme Patrice Maniglier dans sa recherche actuelle sur le structuralisme ${ }^{23}$, notre objectif ne sera pas de trouver « un trait commun » entre l'analyse structurale et la philosophie foucaldienne qui soit transcendant à leur propre dispersion ${ }^{24}$, mais d'explorer des problématiques conceptuelles partagées qui consistent justement en « la matrice de leurs divergences $»^{25}$.

\section{Tel Quel, Nouveau Roman, Nouvelle Critique}

Si, comme l'a affirmé en 1973 Jean Ricardou, - écrivain devenu un des principaux théoriciens de Tel Quel, la revue publiée par les Éditions du Seuil et dirigée par Philippe Sollers entre 1960 et 1982, - le Nouveau Roman n'a pas été une école littéraire ni un groupe ${ }^{26}$, on pourrait peut-être dire qu'il a été l'esprit d'une époque ou même un «moment » de la théorie littéraire naissante en France entre 1950 et 1960. Malgré son hétérogénéité, les auteurs qui ont participé à ce moment avaient en commun au moins la contestation d'un classicisme romantique. «On a besoin d'avant-garde : rien ne rassure plus qu'une révolte nommée », ironisait
Barthes en 1958, tout en affirmant qu'« il n'y a pas d'école Robbe-Grillet $»^{27}$. Pour ce dernier, écrivain polémique et célèbre dès le début des années 1950, l'expression de «Nouveau Roman » était plutôt simplement une « appellation commode $\gg^{28}$. Les désignations courantes d'« École du regard », « Roman objectif », « École de Minuit », «École du Refus $»^{29}$ pour indiquer le Nouveau Roman, vont rassembler un héritage intellectuel et montrer combien il a modifié la manière de comprendre la littérature et le statut de la critique littéraire. Enchevêtrée au Nouveau Roman, l'englobant et le déroutant, se trouve la « Nouvelle Critique littéraire » dont Barthes est le principal nom et qui, au moins après 1963, était aussi très proche de Tel Quel. Il s'agit précisément du mouvement discontinu et fragmentaire de l'«avatar » contemporain du langage, comme le caractérise Foucault dans le texte publié sous le titre de « L'analyse littéraire $» 30$.

De ce fait, même si le nom «Tel Quel » a voulu être une référence à $\mathrm{F}$. Nietzsche ( $\ll$ Je veux le monde et je le veux tel quel $\gg{ }^{31}$ ), rappelons que c'était justement un réalisme réflexif qui était en train d'être contesté par la nouvelle revue. Et Barthes n'aurait pas caché son désaccord : « Le propre du langage, c'est justement de ne pas rendre le monde tel quel! $\gg{ }^{32}$. Contre la critique littéraire exégétique, on assistera à la contestation de plusieurs universaux liés à celle-ci : auteur, génie, œuvre, contexte, époque, mentalité, sens, personnage, sujet. La nouvelle littérature, en faveur d'une démarche non-psychologisante, envisage l'humain et ses émotions en tant qu'effets du texte, et non plus comme des fondements transcendantaux. Dans « L'analyse littéraire », tout comme dans « Les nouvelles méthodes d'analyse

\footnotetext{
Gilles Deleuze, «À quoi reconnaît-on le structuralisme? » (1967), in L'île déserte et d'autres textes, Paris, Éditions de Minuit, 2002, p. 243. Voir notamment : Patrice Maniglier, La Philosophie qui se fait. Dialogue avec Philippe Petit, Paris, Éditions du Cerf, 2019.

4 P. Maniglier, «Introduction », dans P. Maniglier (dir.), Le Moment philosophique des années 1960 en France. Presses Universitaires de France, 2011, pp. 4-33. p. 17

25 Idem.

26 Jean Ricardou, Le Nouveau Roman, Éditions du Seuil, Paris, 1973, p. 6.

27 R. Barthes, «Il n'y a pas d'école Robbe-Grillet », dans Essais Critiques, Paris, Éditions du Seuil, 1964, p. 100.

8 Alain Robbe-Grillet, « À quoi servent les théories » (1955), dans Pour un Nouveau Roman, Paris, Éditions de Minuit, 1963, p. 9

29 A. Robbe-Grillet, «La littérature aujourd'hui », Tel Quel, n 14, 1963, p. 40.

30 M. Foucault, « L'analyse littéraire», in Folie, langage, littérature, op.cit., p. 153

« Déclaration », Tel Quel, n 1, 1960, p. 3-4.

Philippe Forest, Histoire de Tel Quel. 1960-1982, Paris, Éditions du Seuil, 1995, p. 195. Forest mentionne que cette affirmation de Barthes aurait été adressée à Jean-Édern Hallier, se trouvant dans les correspondances dans les archives Hallier.
} 
littéraire », Foucault, donnant suite à ces débats, affirme que le rôle de la critique contemporaine était désormais de se placer au même niveau de l'œuvre commenté : on passe de la critique jugeante (et solennelle) à la critique analysante ${ }^{33}$.

Dans le sillage de E. Allan Poe, P. Valéry, S. Mallarmé, ou de R. Roussel, auteurs-figures « réactivées » dans ces années par plusieurs écrivains, nous sommes alors placés au sein d'une conception du langage dans laquelle l'écrit se dédouble et se représente à soi-même. Pour Foucault, « l'anonymat » du langage a permis à la critique de devenir une « fonction générale sans organisme propre ${ }^{34}$. On aura des procédures basées sur des formes, enveloppant ce qui a été nommé la «bataille de la phrase ${ }^{35}$, la « mise en abîme $\gg^{36}$, « la fin de la représentation », « la trame autour du vide $\gg^{37}$. De cette manière, même si le Nouveau Roman peut être envisagé simplement comme un moment de Tel Quel, cette approche qui mettait l'accent sur les fonctionnalités et ses procédures logiques dans la construction du texte littéraire - décrirait bien les deux projets. Comme idée générale, telle que théorisée par Barthes et en résonance avec la méthode structurale, il y a le déplacement d'une étude des termes (ou des essences) à celle des relations liées par un système. Néanmoins, comme nous allons l'expliciter à partir de la critique de Foucault à Robbe-Grillet, le dépassement philosophique du subjectif par l'objectif comportait de nombreux problèmes : il n'aurait pas fallu choisir l'un à la place de l'autre sous peine de retomber dans les apories (justement contestées par la nouvelle analyse) d'une métaphysique classique.
En ce que concerne le rapport à Barthes ${ }^{38}$, jusqu'à la publication de Folie, langage, littérature ou de La Grande étrangère, on pourrait dire qu'il était un auteur très peu mentionné par Foucault dans l'ensemble de son œuvre. Depuis Le degré zéro de l'écriture, livre de 1953 et en effet cité plusieurs fois par le philosophe dans ces recueils, Barthes nouait définitivement la question de la littérature au problème du langage, qui devrait être traité comme un système de signes. Exactement comme le fera Foucault39, Barthes était en train de poser la littérature comme un objet en soi (« Forme-Objet », « Littérature-Objet »40). Le « degré zéro » est alors le nom de la dernière configuration historique de la littérature, qui affirme la destruction du langage par le biais d'une altération issue d'un nouveau niveau littéraire, celui de l'«écriture » : «l>écriture n>est nullement un instrument de communication, elle n'est pas une voie ouverte par où passerait seulement une intention de langage ${ }^{41}$. C'est à partir de ce « caractère intransitif de l'écriture », comme le désignera Foucault en $1970^{42}$, qu'une séparation entre pensée et langage a pu être également questionnée : le langage demande son propre régime ontologique puisqu'il est irréductible à toutes les autres instances (économique, religieuse, sociale, etc.). L'expérience n'existerait pas de façon « originaire » et en dehors d'un « parler », ou d'un « nommer ». La distance sera alors « la profonde métaphore sur quoi repose tout langage en chemin vers la pensée $\gg^{43}$ en établissant précisément la connexion entre ces deux termes.

Encore dans les années 1950, ces conceptions sur le langage avaient pris leur force également

\footnotetext{
33 M. Foucault, « L'Analyse littéraire », op.cit., p. 156.

M. Foucault, « Les Nouvelles méthodes d'analyse littéraire », in Folie, langage, littérature, op.cit., p 133.

J. Ricardou, Pour une théorie du Nouveau Roman, Éditions du Seuil, Paris, 1971, pp. 118-158.

J. Ricardou, Problèmes du Nouveau Roman, Éditions du Seuil, Paris, 1967, pp. 171-182.

Alain Robbe-Grillet, « Alain Robbe-Grillet au micro de Jean Thibaudeau », programme « Entretiens avec », France Culture, émission du 7 février 1975. Disponible sur : https://www.franceculture.fr/litterature/alain-robbe-grillet-sur-le-nouveau-roman

${ }_{38}$ Foucault parlera de son rapport à Barthes dans une entretien avec Charles Ruas en 1983. Le texte est publié dans les Dits et écris sous le titre «Archéologie d'une passion » (op.cit., vol. 4. pp. 599-608). Cette version diffère néanmoins de celle publiée en appendice à la traduction en anglais de Raymond Roussel (Death and the Labyrinth. The world of Raymond Roussel, Continuum, London/New York. 1986, 2007, pp. 171-188), où Barthes est plus longuement mentionné à la fin.

39 Sur la relation entre Foucault et l'analyse littéraire structurale, nous renvoyons à l'étude de Marie Gil, « Foucault invente l'histoire littéraire », Fabula-LhT, n’ zéro, 2005.

40 Roland Barthes, Le Degré zéro de l'écriture, suivi de Nouveaux Essais critiques, Éditions du Seuil, Paris, 1953, 1972 p. 10-11

${ }_{41}$ Ibid., p. 21. Nous soulignons.

42 M. Foucault, « Folie, littérature, société », in Dits et écrits, vol. 2, p. 114. Nous soulignons

43 M. Foucault, « Guetter le jour qui vient », op.cit., p. 264
} 
dans les livres de Barthes Michelet par lui-même et Mythologies44. Mais c'est à son livre de 1963. Sur Racine, que Foucault fait référence pour introduire l'idée de la «spatialité de l'œuvre »45, enjeu qui débouchera dans l'assignation de sa propre recherche dans le champ de la sémiologie littéraire. En outre, c'est dans Sur Racine que nous rencontrons la question, très importante chez Foucault, de « qu'est-ce la littérature? », ouverte par la réflexion sur « l'être de la littérature $\gg^{46}$. Cette dernière « catégorie » accorde une assignation ontologique propre à la littérature tout en échappant à des présupposés transhistoriques: tout comme la structure, l'être de la littérature se définit par les conditions de sa propre variation ${ }^{47}$. Ce même raisonnement, nous le verrons par la suite, se trouve au cœur du concept d'événement. En tout cas, l'énonciation de cette « question » apparaît chez Barthes pour affirmer une littérature se réfléchissant à partir d'un pli rendu possible par le renversement épistémologique du caractère «second » de la critique : la critique littéraire reprend la valeur d'un langage premier. Comme le démontre Foucault dans la première séance de ses conférences à Bruxelles, « qu'estce la littérature ? »48 nomme en fait l'étendue de l'espace de sa propre possibilité de question et appelle, dans le système qu'elle impose, la reconfiguration de la position de tous les autres termes littéraires à partir de l'auto-référentialité et de l'auto-implication du langage.

Par cette réorientation du langage et par la mise en relief des « tautologies » impliquées dans son fonctionnement ${ }^{49}$, Foucault rejoindra positivement, à plusieurs reprises, le domaine sémiologique. Et cela parce que la sémiologie s'applique à rendre compte du sens tout en contournant une solidification sémantique linéaire ou univoque : les signes rendent possible la signification sans la déterminer préalablement par aucune dynamique
« représentationnelle ». Son Raymond Roussel y insistait déjà : pour chaque noyau de l'œuvre littéraire, pour chaque structure, ou encore pour chaque signifiant, plusieurs signifiés sont possibles. Dans la deuxième conférence à Bruxelles, Foucault propose une « analyse de ces signes par quoi l'œuvre ne cesse de se désigner à l'intérieur d'elle-même »; dans « L'Analyse littéraire » il dit que le langage possède des « réserves indéfinies de sens qui ne sont pas réductibles à des besoins, à des consommations, à des circuits historiquement déterminés $\gg^{50}$. Et il se placera lui-même dans ce courant: «Ne pourrait-on pas faire entrer la littérature dans une sorte de sémiologie générale, propre à une société, et où on l'interrogerait, non pas sur ce qu'elle signifie (les idées, la beauté), mais sur sa structure de signifiant? [...] La littérature, c'est le langage se signifiant lui-même, s'éprouvant dans sa nature de signe [...] $\gg^{51}$.

Après ces considérations, voyons quelques éléments concernant l'enjeu du dépassement du dualisme « objectivité-subjectivité », débat important dans la littérature «avant-gardiste » à ce moment. Dans «Distance, aspect, origine», Foucault dresse une critique à Robbe-Grillet qui cible le caractère trop « objectif » de sa littérature. Par les arguments convoqués, nous remarquerons qu'il s'agit en réalité d'une critique contre le structuralisme - même s'il était en train de le défendre à d'autres moments dans cette période et si l'alternative théorique qui y est proposée reprend, comme nous le montrerons, plusieurs des opérations structurales. En tout cas, vu que le reproche à l'écrivain quant à la place de « l'objet » était courant à l'époque, il ne serait peut-être pas exagéré de rapprocher le ton de la critique foucaldienne à la polémique qui a impliqué Robbe-Grillet et les deux modèles de réalisme ${ }^{52}$. La problématique d'un « réel » pris entre objectivisme et subjectivisme se trouvait

\footnotetext{
44 R. Barthes, Michelet par lui-même, Paris, Éditions du Seuil, 1954 ; R. Barthes, Mythologies, Paris, Éditions du Seuil, 1957.

M. Foucault, « Les nouvelles méthodes d'analyse littéraire », op.cit., p. 139

R. Barthes, Sur Racine, Paris, Éditions du Seuil, 1963, p. 178

R. Barthes, Essais critiques, op.cit., p. 11.

M. Foucault, « Littérature et langage », op.cit., p. 75

M. Foucault, « L'Analyse littéraire », op.cit., p. 166.

Ibid., p. 157

Ibid. p. 166. Nous soulignons.

Voir notamment : Robbe-Grillet, «À quoi servent les théories »; « Temps et description dans le récit d'aujourd'hui » ; « Du réalisme à la réalité » in Pour un Nouveau Roman, op.cit.
} 
au centre des discussions liées à Tel Quel depuis sa naissance, principalement après le colloque organisé par Les Cahiers libres de la jeunesse, en 1960, et qui marque le ralliement de la revue avec Robbe-Grillet ${ }^{53}$. Si ce rassemblement sera bien consolidé et l'écrivain célébré soit avec les

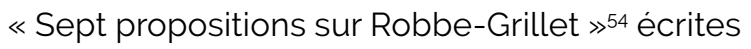
par Sollers, soit avec les éloges de Barthes dans «La littérature, aujourd'hui »55, ou avec le positionnement très favorable de G. Genette dans «Sur Robbe-Grillet $»^{56}$, après la publication de son ouvrage théorique Pour un Nouveau Roman, en 1963, une rupture se met en cours. Du « chosisme » à « l'humanisme », d'une littérature objective et formaliste qui aurait dégénérée en psychologisme existentiel tragique, ou même $d u$ structuralisme à la phénoménologie, tel était le ton de la critique qui faisait apparaître, dans l'espace de quelques années, deux phases bien distinctes de son œuvre. Finalement, en 1964, Sollers écrira, acidement, dans les notes de lecture de Tel Quel : «La quasi-équivalence qui s'est installée avec le temps dans le langage critique de Robbe-Grillet entre les termes subjectif-objectif ne fait que trahir une impossibilité intellectuelle à sortir vraiment du psychologisme [...] Premier temps : théorie "objectale". Second temps : blocage de toute élucidation des significations. Troisième temps: subjectivité absolue [...] »57. De cette manière, encore que les reproches de Foucault à Robbe-Grillet ne portent pas directement sur l'intentionnalité psychologique, sa contestation quant à la nature du vide chez l'écrivain donnera à comprendre justement que la non-maîtrise de l'objet par la trame narrative finissait par transformer cet objet structural dans le vieux sujet. Et cela même s'il ne s'agissait plus d'un sujet en tant que conscience accordée aux personnages, mais d'un objet rendu existant par son emplacement subjectif dans la disposition thématique du récit littéraire. Nous y reviendrons en détail.

\section{Le concept de « réseau »}

Foucault a écrit une seule fois dans Tel Quel (le « Langage à l'infini », sur M. Blanchot); a participé au colloque « Une littérature nouvelle? » organisé par la revue ; a publié un compte rendu sur Les Aventures d'une jeune fille de Jean-Edern Hallier («Un "Nouveau Roman" de terreur ») ; a discuté la possibilité d'un projet éditorial avec les Éditions du Seuil - tout cela en 1963, la même année de publication de Raymond Roussel. En avril 1964. le philosophe publie « Le langage de l>espace », recueil de notes sur les travaux de quelques écrivains liés aussi à Tel Quel, Roger Laporte, Jean-Marie Le Clézio, Claude Ollier et Michel Butor ; et, en 1966, il écrit un compte-rendu sur Jean Thibaudeau, intitulé « $\dot{A}$ la recherche du présent perdu $\gg^{58}$. «Distance, aspect, origine », article paru chez Critique en novembre 1963, n'est pas simplement le grand éloge du projet tel quelien par Foucault, mais configure, avec l'ensemble des textes que nous mentionnons, le développement d'une théorie originale de critique littéraire. Celle-ci, dans ce texte, se révèle à partir de sa lecture de Robbe-Grillet, qui peut désormais être désigné comme étant le « motif » du réseau Tel Quel. Réseau, parce que l'écrivain est traité par Foucault comme objet de comparaison, de juxtaposition, de contestation ; et c'est précisément par ce complexe croisement des usages que sa littérature a pu fonctionner comme un motif dans le texte foucaldien. L'article intercalera alors des descriptions spécifiques des livres de Robbe-Grillet, de Sollers, de Jean-Louis Baudry, de Marcelin Pleynet et de Jean Thibaudeau avec des analyses sur le rôle du langage, en faisant retourner constamment, dans un mouvement de décollement de la fonction critique, le plan de la théorie vers celui de la pratique littéraire.

En ce qui concerne alors le « motif » Robbe-Grillet, c'est le caractère de critique élogieuse

\footnotetext{
53 Ph. Forest, Histoire de Tel Quel 1960-1982, op.cit., p. 70.

54 Ph. Sollers, «Sept propositions sur Robbe-Grillet », Tel Quel, n² 2, 1960.

55 R. Barthes, « La littérature, aujourd'hui », Tel Quel, n 7, 1961.

Gerard Genette, «Sur Robbe-Grillet », Tel Quel, n 8, 1962.

Ph. Sollers, «A. Robbe-Grillet: Pour un Nouveau Roman», Tel Quel, n 18, 1964, p. 93-4.

58 M. Foucault, «Langage à l'infini », Tel quel, no 15, automne 1963 (Dits et écrits, vol. 1, pp. 250-261) ; « Un "Nouveau Roman" de terreur », France-Observateur, $14^{\text {eme }}$ année, $n^{\circ} 710,12$ décembre 1963 (Dits et écrits, vol. 1, pp. 285-287); « Le langage de l>espace », Critique, $n^{\circ} 203$, avril 1964 (Dits et écrits, vol. 1, pp. 407-412) ; « À la recherche du présent perdu », L'Express, n 775, 25 avril - $1^{\circ}$ mai 1966 (Dits et écris, vol. 1, pp. 504-505).
} 
qui rend l'écrit foucaldien assez double. L'auteur de La Jalousie 59 parcourt l'article comme déclencheur d'autres problématiques, dont la principale serait celle concernant la question dialectique de l'opposition entre l'objectif et le subjectif. Quant à sa forme, la nouvelle critique foucaldienne traitera les œuvres, ici sous l'égide de Tel Quel, par une « articulation discursive ». Celle-ci érige le « réseau » en concept littéraire en l'éloignant d'une méthode exégétique par la manière même dont se fera la liaison entre ces auteurs : «Il y a peut-être là, d'une œuvre à l'autre, l'image, non d'une mutation, non d'un développement, mais d'une articulation discursive ; et il faudra bien un jour analyser les phénomènes de ce genre dans un vocabulaire qui ne soit pas celui, familier aux critiques [...] des influences et des exorcismes $\gg^{60}$. Le plan résultant d'un tel entrecroisement est celui d'une diagonale, l'espace crée par ce que nous voyons se constituer comme la distance générale entre les auteurs en particulier.

Dans « Débat sur le roman », transcription d'une des tables rondes du colloque organisé par Tel Quel, Foucault, qui dirigeait la séance, a évoqué « une cohérence très évidente » chez le groupe, en affirmant que ce qui le rapprochait d'eux était une manière de concevoir l'expérience ${ }^{61}$. Comme la rencontre se déroulait dans le moment d'éloignement de la revue par rapport à Robbe-Grillet, Foucault désignera l'écrivain comme étant « une cache, un point aveugle, quelque chose à partir de quoi on parle et qui n'est jamais là $\gg^{62}$. Cette dimension de «sol » ou d'étendue se faisait explicite depuis son affirmation de que, différemment du psychologisme surréaliste, c'était la dimension du « voir et parler » en tant qu'ex- périences de la pensée, qui avait été ouverte par les œuvres de Roussel et de Robbe-Grillet ${ }^{63}$. Déjà dans l'article de Critique, référé comme un «Il majeur » et comme la «possibilité d'existence » ${ }^{64}$ pour la production de Tel Quel, Robbe-Grillet va progressivement, sous la plume de Foucault, perdre son individualité pour devenir le point de convergence d'une intertextualité et instrument pour l'application de sa propre théorie. Le « réseau » peut alors être envisagé comme un motif structural chez Foucault, étant l'une des figures du structuralisme que le philosophe reprend pour transformer en outil méthodologique :

\begin{abstract}
Si j'ai tenu à ces références à Robbe-Grillet, un peu méticuleuses, c'est qu'il ne s'agissait pas de faire la part des originalités, mais d'établir, d'une œuvre à l'autre, un rapport visible et nommable en chacun de ses éléments et qui ne soit ni de l'ordre de la ressemblance lavec toute la série de notions mal pensées, et à vrai dire impensables, d'influences, d'imitation) ni de l'ordre du remplacement (de la succession, du développement, des écoles) : un rapport tel que les œuvres puissent s'y définir les unes en face, à côté et à distance des autres, prenant appui à la fois sur leur différence et leur simultanéité, et définissant, sans privilège ni culmination, l'étendue d'un réseau ${ }^{65}$.
\end{abstract}

Le concept de « réseau », en tant que figure liée au «système », était présent chez le philosophe depuis le début des années 1960, dans Histoire de la folie à l'âge classique ou dans Naissance de la clinique. Dans le contexte littéraire, on le perçoit dans Raymond Roussel, dans « Le langage à l'infini », « Postface à Flaubert », « Le Mallarmé de J.-P. Richard $\gg^{66}$, entre autres textes ; après 1965, il apparaîtra dans le cadre des Mots et les choses. Suivant nos considérations sur l'analyse littéraire structurale, nous voyons que

\footnotetext{
59 A. Robbe-Grillet, La Jalousie, Paris, Les Éditions de Minuit, 1957.

60 M. Foucault, «Distance, aspect, origine », Critique, n 198, novembre 1963 (Sur J.-L. Baudry, Les Images, Paris, Éd. du Seuil, 1963 ; M. Pleynet, Paysages en deux : les lignes de la prose, Paris, Éd. du Seuil, 1963 ; P. Sollers, L'Intermédiaire, Paris, Éd. du Seuil, 1963, et Tel Quel, n 1-14, 1960-1963), repris dans Dits et écrits, vol 1, op.cit., pp. 272-285, ici p. 273.

${ }_{61}$ M. Foucault, « Débat sur le roman », Tel Quel, no 17, printemps 1964 (Cerisy-la-Salle, septembre 1963 ; débat organisé par le groupe de Tel Quel sur le thème « Une littérature nouvelle? », session dirigé par M. Foucault, avec G Amy, J.-L. Baudry, M-J. Durry, J.P. Faye, M. de Gandillac, C. Ollier, M. Pleynet, E. Sanguineti, P. Sollers, J. Thibaudeau, J. Tortel), repris dans Dits et écrits, vol. 1, op.cit., pp. 338-390, ici p. 338. 62 Ibid., p. 367.

63 M. Foucault, « Distance, aspect, origine », op.cit., p. 339. Ce même rapprochement apparaît dans « La littérature et la folie - Raymond Roussel », in Folie, langage, littérature, op.cit., pp. 111-125.

64 M. Foucault, « Distance, aspect, origine», op.cit., p. 273. L'auteur souligne.

65 Ibid., p. 278. Foucault souligne.

66 M. Foucault, «Le langage à l'infini », op.cit., p. 256 ; «Sans titre », Postface à Flaubert (G), Die Versuchung des Heiligen Antonius, Francfort, Insel Verlag, 1964 (Dits et écrits, vol. 1, p. 298); « Le Mallarmé de J.-P. Richard », Annales. Économies, sociétés, civilisations, n 5. septembre-octobre 1964 (Dits et écrits, vol. 1, p. 429).
} 
le « réseau » est ici introduit pour rendre possible parler de l'auteur tout en évitant le plan interprétatif. Le réseau caractérise la disposition systématique des structures littéraires, le mouvement anonyme du langage et même la fonction de la critique. Dans « Les Nouvelles méthodes de l'analyse littéraire » on lit justement: «La critique, c'est le réseau que le langage trame spontanément et sans cesse entre chacun de ses points. Ce n'est plus une instance de décision, c'est une forme de coexistence $\gg^{67}$. Le réseau libère le commentaire de la nécessité d'être le décalque propre à une reconnaissance ou à une explication du texte premier. Comme l'affirme la note du 14 décembre 1963 du cahier $\mathrm{n}^{\circ} 4$ de son Journal intellectuel, la pensée du « réseau » engage l'expérience contemporaine du langage une fois que la « critique » - cette «poursuite formelle »- doit elle-même opérer par une «mise en constellation $»^{68}$. Spatial - et nous lisons, structural -, le réseau est ainsi le connecteur qui permet une analyse non-causale, qui soit de l'ordre des discours et des énoncés disposés en séries, et non des thématiques, des contenus et des objets circonscrits au préalable: c'est le réseau qui crée la donnée littéraire. Nous « mélangeons » le vocabulaire de L'Archéologie du savoir volontairement : même si la notion de «formation discursive » telle qu'elle apparait dans l'archéologie subséquente va ouvrir un espace plus formalisé que le réseau ou les articulations ici encore rattachés plutôt à la littérature, il y a une forte ressemblance entre ces analyses de coupure transversale dans la mesure où elles s'appuient toutes sur un modèle systématique de communication entre concepts.

\section{La « lacune » de Robbe-Grillet : isomorphisme, mise en abîme, volume satellite}

La problématique de l'espace ouverte par l'idée d'isomorphisme (présente aussi dans Naissance de la Clinique, et avec d'autres contours, dans les épistémès des Mots et les choses) sera ce qui permettra, dans «Distance, aspect, origine », la particulière communication de toutes ces œuvres liées, en réseau, à Tel Quel. La notion d'isomorphisme établit un fort dialogue avec les méthodes comparatives - qu'on pense à Georges Dumézil ou à Claude Lévi-Strauss ${ }^{69}$. Dans l'article, les isomorphismes, écrit Foucault, «ne sont pas des "visions du monde", ce sont des plis intérieurs au langage ; les mots prononcés, les phrases écrites passent par eux, même s'ils ajoutent des rides singulières $\gg{ }^{70}$. Accouplé à cet isomorphisme, on aura aussi le concept de mise en abîme, tellement utilisé par la littérature associée au Nouveau Roman : «Il me semble [...] que les possibilités du langage à une époque donnée ne sont pas si nombreuses qu'on ne puisse trouver des isomorphismes (donc des possibilités de lire plusieurs textes en abîme) [...] $\gg^{71}$. Dans le contexte dont nous nous occupons ici, ces isomorphismes sont mentionnés par le philosophe également dans « Débat sur le roman » (en référence à un terrain commun entre Tel Quel et les surréalistes à l'égard de « l'expérience », mais à partir duquel il va pouvoir diagnostiquer justement leur divergence), et dans « Débat sur la poésie » (pour situer son propre travail en « résonance » avec celui de la revue ${ }^{72}$. Dans la conférence « Structuralisme et analyse littéraire »,

\footnotetext{
67 M. Foucault, « Les nouvelles méthodes d'analyse littéraire », op.cit., p. 134. Nous soulignons.

68 Le Journal intellectuel désigne l'ensemble des vingt carnets personnels de Foucault, réunis dans les boîtes XCl et XCll du Fonds Foucault (BnF, NAF 28730). Pour cette citation : Foucault, Journal intellectuel, boîte XCl, cahier n 4 , note du 14 décembre 1963 . À propos du statut du Journal intellectuel dans la recherche actuelle sur l'œuvre foucaldienne, voir : Ph. Sabot, « Dans les "Archives" de l'Archéologie. Relire Les Mots et les choses aujourd'hui », Rev. Filos. Aurora, Curitiba, v. 28, n 45, pp. 747-766, set/dez 2016.

69 Des discussions à propos de la recherche de Lévi-Strauss apparaîtront à plusieurs endroits dans Folie, langage, littérature, parfois entrecroisées à la problématique de l'analyse littéraire. Voir notamment : « Folie et civilisation », p. 29 ; « Folie et civilisation. Conférence prononcée au Club Tahar Haddad à Tunis en avril 1967 », p. 54 ; « Les nouvelles méthodes d'analyse littéraire », p. 144 ; « L'Analyse littéraire », p. 165. Dans « Structuralisme et analyse littéraire », Lévi-Strauss sera rapproché de Dumézil (p. 201-202, op.cit.).

70 M. Foucault, « Distance, aspect, origine », op.cit., p. 274.

71 Ibid., p. 273. Nous soulignons.

72 M. Foucault, « Débat sur la poésie », Tel quel, n 17, printemps 1964 (Cerisy-la-Salle, septembre 1963 ; débat organisé par le groupe de Tel quel avec M. Foucault, J.-L. Baudry, M.-J. Durry, J. P. Faye, M. Pleynet, E. Sanguineti, Ph. Sollers, J. Tortel), repris dans Dits et écrits, vol. 1, pp. 390-406, ici p. 395.
} 
la figure servira de contraposition à la logique de la « causalité » en littérature ${ }^{73}$. L'isomorphisme englobe alors non pas seulement les ressemblances entre les éléments étudiés, mais aussi bien - et surtout - les différences ; il est, dans ce sens, la figure irréductible de leur connexion. Et c'est de cette manière que l'isomorphisme touche au système et à la structure et, par conséquent, donne à voir l'événement.

En consultant ses notes de la même année dans Le Journal intellectuel, on s'aperçoit que la figure de l'isomorphisme est également à l'œuvre pour penser les « formations culturelles » (problématique qui reviendra, avec d'autres contours, par l'analyse des travaux de Lévi-Strauss dans l'inédit Cours de Tunis ${ }^{74}$, de 1966-67). Même si cette notion relève dans ce cahier $n^{\circ} 3$ d'une discussion autour des « expériences-limites $\gg^{75}$, nous croyons, néanmoins, que l'isomorphisme tel qu'il y est décrit peut éclaircir notre problématique autour de l'analyse littéraire par son statut de moteur de comparaison : « l'isomorphisme», écrit Foucault, est un «principe », et non un « fait »; en tant qu'outil épistémologique, il doit servir à conduire « l'ordre systématique qu'il faut constituer » dans l'étude ${ }^{76}$. C'est la méthodologie propre à la coupure qui est donnée par l'isomorphisme, en lui accordant le caractère « fictif » d'instrument heuristique. Le philosophe énumère alors les «principes » par lesquels l'analyse des formations culturelles devrait procéder, à savoir, « Le corps impalpable », «L'arbitraire de la coupure » et « La clôture et la récurrence » - le premier signalant que la formation culturelle n'est pas une chose. mais un complexe «multilinéaire », et les deux derniers renvoyant au fonctionnement même du système. Il décrit ainsi une procédure qui met ensemble deux perspectives : l'assignation de la «structure d'événement » et la « critique de la récurrence ». Dès lors, le « principe » de l'isomorphisme est au plus près de la structure : l'isomorphisme est ce à partir de quoi l'événement peut apparaître, il est ce qui donne à voir l'événement. La structure ne s'oppose pas à l'événement justement parce qu'elle se compose non pas d'éléments fixes mais d'actualisations permanentes. Dans cette note, on lit:

\begin{abstract}
2 ème principe : l'arbitraire de la coupure. En principe en tout point de la chaîne temporelle on peut établir une coupure : et une certaine correspondance se découvrira entre les différentes lignes [...]. Ce qui revient à dire que l'isomorphisme n'est pas un fait, un événement secret sur lequel on pourrait tomber, ou qu'on pourrait aussi bien ne pas découvrir. L'isomorphisme est un principe général des formations culturelles: on le trouvera partout [...] plus ou moins facile à repérer, assignable à tel ou tel niveau. Mais cela ne veut pas dire que l'événement disparaît au profit de la structure: on pourrait aussi bien dire, et mieux peut-être, que tout à chaque instant est, de droit, et par [structuration*], un événement. II s'agit d'une perception indéfiniment événementielle ${ }^{77}$.
\end{abstract}

Comme dans la méthode comparative, c'est à la loi théorique (la structure) de comporter sa propre variation, le changement (multi-causal) se rend visible seulement à partir du système qui l'organise - c'est la structure qui rend possible le repérage de l'événement. Par la suite, encore dans ce même cahier, on aura également une définition de «structure » : « ensemble d'opérations à contenu indéfini $\gg^{78}$. D'une façon générale, on assiste alors à un enchevêtrement entre structure et événement à partir d'une archéologie conçue pour être plus fondamentale que le structuralisme, bien qu'à lui lié par un similaire modus operandi de ses nouveaux concepts. Et si on situe cette figure épistémologique dans la méthode structurale, on comprendra que loin d'avoir pour fonction d'assigner une quelconque prévisibilité ou déterminisme, les isomorphismes - organisés en tableaux ${ }^{79}$ - sont ce qui garantit une analyse immanente des éléments étudiés, étant donné

\footnotetext{
M. Foucault, «Structuralisme et analyse littéraire. Conférence prononcée au Club Tahar Haddad à Tunis le 4 février 1967 », op.cit., p. 204

M. Foucault, Cours de Tunis, BnF, Fonds Foucault, NAF 28730, boîte LVIII, dossier 1.

M. Foucault, Journal intellectuel, op.cit, boite XCl, cahier $n^{\circ} 3$, note du 4 juin 1963. Dans cette note nous lisons aussi une « liste des formations culturelles » : « la mort, la décadence, l'aveu, la sexualité, la folie ».

Ibid., note du 28 mai 1963. Nous soulignons.

Ibid. Mot difficilement lisible, celui de «structuration » nous semble le plus proche.

M. Foucault, Le Journal Intellectuel, op.cit., boîte XCl, cahier n 3, note du 21 juin 1963.

M. Foucault, « Distance, aspect, origine », op.cit., p. 273.
} 
qu'il n'y a pas d'intelligibilité explicative surplombante et qui ne vienne que des relations qui définissent l'identité même de ces éléments. L'isomorphisme - cette répétition par corrélation qui entraîne simultanément ses propres différences - est, de la sorte, une composante majeure du fonctionnement de la structure.

À côté de l'isomorphisme littéraire, nous avons souligné dans « Distance, aspect, origine »le concept de « mise en abîme ». Même si l'expression pourrait remonter, en littérature, comme rappelle Ricardou, au Journal d'André Gide de $1893^{80}$, ici on a plutôt affaire à la démarche que le théoricien a décrit dans son Problèmes $d u$ Nouveau Roman, de 1967, lorsqu'il associe la mise en abîme à une dramatisation du récit par lui-même, qui a pour conséquence une abolition du temps ${ }^{81}$. Cette dramatisation de la forme est faite par des pliures dans le roman, avec les plusieurs couches d'une histoire qui se répète, à chaque fois remplissant une lacune présente dans la série précédente et ouvrant sur une nouvelle lacune: c'est le fonctionnement du « sériel » en analyse structurale. La mise en abîme se fait alors importante pour comprendre le statut particulier de ce que Foucault désignera, dans cet article, comme le « retour » du même au travers des « analogies ». Il s'agit d'un même qui ne s'oppose pas à la différence, mais dont la répétition fait encore dissoudre le contenu factuel de l'intrigue. Sur le roman Une Cérémonie royale, de Thibaudeau, le philosophe écrit :

Là, les partages entre réel et virtuel [...] n'ont plus d'autre valeur que d'être moments du passage, relais plus que signes, traces de pas, plages vides où ne s'attarde pas mais par où s'annonce de loin, et s'insinue déjà, ce qui d'entrée de jeu était le même (renversant à l'horizon, mais ici même également en chaque instant, le temps, le regard, le partage des choses et ne cessant d'en faire paraître l'autre côté). [...] [Il s'agit d'un] mouvement presque sur place, [d'une] attention recueillie à l'Identique [...] ${ }^{82}$.
Associé à l'isomorphisme et à la mise en abîme, Foucault forgera ensuite le concept de « volume satellite », image heuristique vidée d'épaisseur et qui sert à qualifier les figures présentes dans Parc, Une Cérémonie royale ou dans Images, respectivement les livres de Sollers, de Thibaudeau et de Baudry. Le volume satellite conjure, par la multiplication de l'identité, la présence et l'absence des objets thématiques qui ne peuvent non plus être appréhendés ou définis par son contenu, mais seulement par leur position mouvante, sagittale ${ }^{8_{3}}$ dans le système littéraire de la narrative où ils sont placés. Le volume, « l'intérieur des objets à l'extérieur d'eux-mêmes $»^{84}$, est alors topologique parce qu'il pousse les contenus narratifs à être toujours en train de se défaire aussitôt que leurs contours viennent à apparaître, ou à se produire. Il s'agit d'un mouvement qui, en arrivant au point de cristallisation des faits narratifs, les efface. Mais, finalement, toutes ces descriptions des travaux des écrivains, utilisées par le philosophe positivement et proche de sa propre théorie, sont en fait mises en contraste négativement à l'égard de Robbe-Grillet :

Sans doute, certaines figures [...] du Parc, d'Une cérémonie royale ou des Images sont-elles sans volume intérieur, allégées de ce noyau sombre, lyrique, de ce centre retiré mais insistant dont Robbe-Grillet déjà avait conjuré la présence. Mais, d'une manière assez étrange, elles ont un volume [...] ces volumes satellites [...] ne manifestent de la chose ni sa présence ni son absence, mais plutôt une distance qui tout à la fois la maintient loin au fond du regard et la sépare incorrigiblement d'elle-même ${ }^{85}$;

On pourrait dire que si, chez Robbe-Grillet, les choses s'entêtent et s'obstinent, chez Sollers elles se simulent [...] $]^{86}$;

Les spectateurs chez Robbe-Grillet sont des hommes debout et en marche [...] ils ont déjà pénétré au milieu de ces choses qui se présentent à eux de profil, tournant à mesure qu'ils les contournent. Les personnages du Parc, des Images sont assis, immobiles, en des régions un peu décrochées de l'espace [...] Régions séparées, mais par quoi ? Par rien

\footnotetext{
J. Ricardou, Problemes du Nouveau Roman, op.cit., p. 172 Ibid., pp. 173-188.

M. Foucault, « Distance, aspect, origine », op.cit., p. 277. Nous soulignons.

Foucault, «Distance, aspect, origine », p. 276

Ibid., p. 274.

Ibid., p. 274

36 Ibid., p. 275
} 
d'autre sans doute qu'une distance [...] que rien ne peut résorber, ni meubler [...] $]^{87}$.

Mais d'où viendrait ce décalage ? Pourquoi l'arrangement conceptuel forgé par Foucault ne pourrait pas être appliqué à l'écrivain ? Ne serait-ce pas du même principe de « volume satellite » foucaldien duquel parlait Robbe-Grillet, puisqu'il a tant affirmé que dans son Nouveau Roman le « récit tournait autour d'un vide ${ }^{88}$ ? Des Gommes ${ }^{89}$, par exemple, l'auteur a affirmé qu'il s'agissait d'un roman policier « dont le crime était absent » ; du roman Le Voyeur ${ }^{\circ}$, que c'était le développement minutieux de vingt-quatre heures d'un voyage, à l'exception d'une heure, exactement le seul espace de temps « où s'est peut-être passé quelque chose »; de La Jalousie que c'était un récit fait par « un narrateur qui est lui-même absent de son récit $\gg^{91}$. Le « creux », le «manque », l'absence, - ou, ajoutons-nous, la « case vide $\gg^{92}-$, seraient tous également des catégories convoquées par sa littérature pour désigner les brèches et les écarts entre les os du squelette du roman laissés par l'auteur à l'usage libre d'un lecteur maintenant appelé à l'action, et qui autrefois étaient remplis par tant de sentiments, des faits ordonnés, de plein, de chair. Mais là il ne faut pas pourtant que l'image du squelette nous trompe : en réalité il n'y a pas quelque chose comme une opposition entre forme et conten ${ }^{93}$, mais une pensée dans laquelle leur interdépendance est rappelée pour empêcher soit une quelconque systématicité facile par rapport à la chronologie narrative, soit une naturalisation interprétative ${ }^{94}$. Ou bien il ne devrait pas y avoir une telle disjonction, une telle limite assignée.
Ainsi, dès lors, malgré la ressemblance entre ces pensées formelles sur le « creux», c'est exactement à propos de la place du «vide » (ou même sur la nature heuristique du vide) que Foucault critiquera Robbe-Grillet :

De toutes parts, on reconnait [...] le vide essentiel où le langage prend son espace; non pas lacune comme celles que le récit de RobbeGrillet ne cesse de couvrir, mais absence d'être, blancheur qui est, pour le langage, paradoxal milieu et aussi bien extériorité ineffaçable. $L a$ lacune n'est pas, hors du langage, ce qu'il doit masquer, ni, en lui, ce qui le déchire irréparablement. Le langage, c'est ce vide, cet extérieur à l'intérieur duquel il ne cesse de parler [...] ${ }^{95}$.

Le philosophe lui opposera ainsi, point par point, les autres écrivains analysés dans l'article - tout en déployant, simultanément, une critique du structuralisme. Nous la comprenons par une séparation des concepts en deux blocs, l'un correspondant à sa propre théorie (et qu'il repère chez - ou en décalque de - Sollers, Thibaudeau, Baudry et Pleynet) et l'autre correspondant à Robbe-Grillet et à l'analyse structurale. Ainsi, dans le premier bloc, la méthode procède par la « multiplication des identités », « retour du Même », « distance » vidée, repérage des « isomorphismes à travers d'une épaisseur d'analogies », événement traité comme « recueillement de l'identique ». Dans le deuxième, ily a « lacune » trop peuplé par l'objet, « déchiffrement des signes à travers un système de différences », « lecture d'événement $\gg^{96}$. La nouvelle analyse foucaldienne, au-delà de la structure et du signe, serait donc une complexification de l'interdépendance des concepts de même et de différence qui puisse ouvrir à une pensée non-dualiste entre sujet et objet, c'est-à-

\footnotetext{
${ }^{87}$ Idem. Nous soulignons.

88 A. Robbe-Grillet, « Alain Robbe-Grillet au micro de Jean Thibaudeau », op.cit.

A. Robbe-Grillet, Les Gommes, Paris, Éditions de Minuit, 1953.

A. Robbe-Grillet, Le Voyeur, Paris, Éditions de Minuit, 1955.

A. Robbe-Grillet, « Alain Robbe-Grillet au micro de Jean Thibaudeau », op.cit.

Il s'agit du « sixième critère » évoqué par Deleuze dans « À quoi reconnaît-on le structuralisme ? », op.cit., p. 258-265.

En 1961, dans «Qu'est-ce la littérature aujourd'hui ? », Barthes affirmait que son formalisme structural ne s'appuyait pas sur une division entre forme et contenu, mais se concentrait plutôt sur la signification : « La signification, c'est-à-dire : l'union de ce qui signifie et de ce qui est signifié ; c'est-à-dire encore : ni les formes ni les contenus, mais le procés qui va des uns aux autres », dans Essais Critiques, op.cit., p. 155. Nous soulignons. Foucault, dans «L'Analyse littéraire », a également insisté sur l'enchevêtrement des deux termes (op.cit., p. 160). 94 Comme l'affirme P. Maniglier dans « Du mode d>existence des objets littéraires : enjeux philosophiques du formalisme », « Les formes sont les seuils d'objectivation de la littérature. Le formel n'est donc pas moins que le matériel, comme si l'on pouvait l'obtenir par soustraction (en ne tenant pas compte du thème, du sens, etc.) ; il est plus. La forme est cette part en excès qui fait dériver une expérience malgré nous et qui existe dans cette dérive même », Les Temps Modernes, n 676, 2013, pp. 48-80, p. 57.

95 M. Foucault, « Distance, aspect, origine », op.cit., p. 284. Nous soulignons.

96 Ibid., p. 277.
} 
dire, sans accorder de privilège à aucun des deux. Il s'agissait d'un dépassement de la dialectique que l'analyse structurale n'aurait pas accompli parce qu'elle aurait privilégié l'objet, et, avec lui, l'assignation figée de la « différence » : le contenu était soumis à l'empire de la forme.

Par conséquent, le «volume satellite » foucaldien, mais encore plus sa notion de « distance », vient exactement s'opposer au vide de Robbe-Grillet (et du structuralisme). Ce vide était trop annoncé, trop placé, configurant le centre insistant 97 qui isolerait « deux parts du monde: un sujet et un objet ou les choses en face de la pensée $\gg^{98}$. L'écrivain aurait ainsi transféré à l'objet ce qui avait été libéré du côté du « vieux » sujet fondateur ; dans l'envie de dépsychologiser, le structuralisme aurait tout simplement substitué les notions traditionnelles par les déterminismes de certains termes, comme le système, la différence, les signes, etc. Mais on reconnaît la critique, elle est adressée plusieurs fois à la notion de structure : si la lacune créée pour contourner l'emplacement du sujet redevient présence, la métaphysique refoulée fait retour, et la structure ne serait qu'un autre nom pour l'ancien fondement. Contrairement à cette approche, affirme Foucault dans l'article, dans le bloc formé Sollers, Baudry, Pleynet, Thibaudeau - et lui-même -, il n'y a pas de présence de la lacune, mais plutôt de la « distance », qui ne sépare pas, mais ouvre. Et cela «non pour en faire cohabiter les formes successives en un espace de parcours (comme chez Robbe-Grillet), mais pour les laisser venir plutôt dans une dimension sagittale $» 99$. Ce «monde de la distance » pourra alors être celui de «l'identité buissonnante, du Même au point de sa bifurcation, ou dans la courbe de son retour $\gg^{100}$.

\section{Événement, aspect, expérience}

Du côté de la théorie défendue par Foucault, dont les traits nous rassemblons donc sous le « premier bloc », le « retour du Même » désigne une bifurcation et un décalage de soi qui garantissent que le contenu positif de la narrative sera effacé, certes, mais cette-fois dans un mouvement qui ne prendra ni le même ni la différence de forme ontologique et placée, mais de forme circulaire (démarche non accomplie par Robbe-Grillet, ni apparemment ici par le structuralisme, qui auraient transformé le vide dans une chose, un objet solidifié dans l'intrigue). Tout cela modifie finalement le statut de l'événement tel qu'il est annoncé dans le texte, scindé qualitativement en deux usages opposés : soit événement comme « lecture » (Robbe-Grillet et l'analyse structurale) ; soit événement virtuel en tant que « recueillement de l'identique » (caractère décalqué de Thibaudeau). La Jalousie, Le Voyeur et La Chambre secrète étant des livres de Robbe-Grillet, nous pouvons visualiser plusieurs des oppositions entre les deux blocs dans ce passage, bien comme la nouvelle configuration de l'événement:

\begin{abstract}
Étalé à côté de lui-même, le temps de $L a$ Jalousie et du Voyeur laisse des traces qui sont des différences, donc finalement un système de signes. [...] chez Robbe-Grillet, la différence entre ce qui a eu lieu et ce qui n'a pas eu lieu même si (et dans la mesure où) elle est difficile à établir, demeure au centre du texte (au moins sous forme de lacune, de page blanche ou de répétition): elle en est la limite et l'énigme ; dans La Chambre secrète, la descente et la remontée de l'homme le long de l'escalier jusqu'au corps de la victime [...] est après tout la lecture d'un événement. Thibaudeau [...] semble suivre un dessin semblable : en fait, il s'agit [...] de déployer une série d'événements virtuels [...] loù on] ne déchiffre pas de signes à travers un système de différences; on suit des isomorphismes, à travers une épaisseur d'analogies. Non pas lecture, mais plutôt recueillement de l'identique, avancée immobile vers ce qui n'a pas de différence ${ }^{101}$.
\end{abstract}

L'«événement virtuel », opposé donc à « l'événement-lecture », est ce qui arrive et est entre, logé dans l'écart, dans la distance : l'événement sous l'égide d'une « répétition » circulaire et non différée. La distance étale l'événement en ne lui permettant aucune substantialisation, en faisant

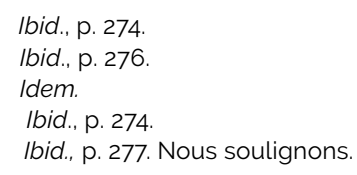


que l'événement ne se corporise dans aucun « centre ». L'événement virtuel n'occupe pas une place démarquée par quelconque « objet » et ni même par le « contours » issu du vide de l'objet. L'assignation par Foucault d'un « événement-lecture » chez Robbe-Grillet dénonce alors l'emplacement figé des faits dans ses récits, même s'ils étaient dépourvus de contenu chronologique/historique: «lecture » parce que la place de l'événement serait tellement marquée qu'elle finissait par l'essentialiser.

Néanmoins, les arguments que le philosophe utilise comme contrepoint à la démarche de Robbe-Grillet et du structuralisme suivent curieusement le même raisonnement structural - l'enchevêtrement du même dans la différence et vice-versa ${ }^{102}$-, ce qui fait que son affirmation à propos de l'insistance structurale sur la soi-disante fixité immobile de la « différence » devienne caricaturale. Ces débats méthodologiques en effet se trouvent proches de ceux sur le « centre de la structure » ou ceux sur le « kantisme sans sujet transcendantal $»^{103}$, en tant qu'ils ciblaient aussi une reconduction latente de la place du « sujet » dans l'analyse structurale. Néanmoins, c'est bien la structure qui se trouve en mesure de conduire l'interrogation sur la cohérence transhistorique des objets philosophiques ou sur le statut de celui qui les énonce : c'est tout l'enjeu de la reconfiguration des modes de détermination de l'« identité » qui prenait essor dans la linguistique et dans la sémiologie modernes. De plus, Foucault modifie constamment ses positions. Si dans cet article de 1963 le « signe » est la dimension structurale critiquée chez Robbe-Grillet, dans le colloque de Tel Quel à Cerisy-la-Salle c'est le philosophe qui affirme la centralité de la notion en disant qu'elle était « au milieu » de leur débat et en proposant que la discussion se poursuive autour du « champ du signifiant et du signifié $»^{104}$.

En tout cas, la notion « d'événement virtuel » est aussi ce qui pourra rendre compte des autres termes qui figurent dans le titre de l'article, l'aspect et l'origine. Par un réaménagement théorique, Foucault met en place une critique litteraire où la catégorie de « l'espace » sera substituée par l'axe de la « distance », en même temps que la catégorie grammaticale de « l'aspect » viendra à la place du « temps ». Mais cette notion d'« aspect » correspond, en linguistique, à une indication sur le déploiement de l'action, qui est étudiée d'un point de vue interne au prédicat. Ainsi l'aspect s'opposera au temps verbal, puisque celui-ci est toujours référé de l'extérieur de l'action. Cette problématique, qui remonte au linguiste Gustave Guillaume, a été retravaillée dans les années 19501960 par Paul Imbs, qui insistait sur la question du « déroulement » interne dans la description des actes ${ }^{105}$. Dans le Dictionnaire Encyclopédique des sciences du langage, de 1972, O. Ducrot et T. Todorov expliquent à propos de l'aspect:

\begin{abstract}
Une distinction [séparera] [...] les indications temporelles au sens strict, c'est-à-dire les indications chronologiques, visant à une datation [les temps verbaux], et les indications d'aspect. Celles-ci sont toujours intérieures au prédicat. Le prédicat comporte en effet, non seulement l'idée d'une certaine qualité ou d'une certaine action (《être bleu », « venir dîner »), mais l'idée d'un certain mode de manifestation dans le temps de cette action ou de cette qualité, l'indication de la façon dont elles remplissent la période concernée par l'énonciation ${ }^{106}$.
\end{abstract}

Il faut alors encore indiquer l'autre perspective que l'aspect offre : l'accent mis sur le déroulement interne de l'action devient une technique pour réussir à élider le sujet de l'énonciation : sorte d'énoncé grammatical qui s'énonce à soi-même, l'aspect fait référence seulement à l'action, et non à la perspective du sujet qui fait l'analyse (le

\footnotetext{
102 Nous pensons certainement à Différence et répétition et à Logique du sens de Deleuze, qui ont complexifié ce dualisme, mais qui sont pourtant publiés quelques années plus tard, en 1968 (Paris, P.U.F) et 1969 (Paris, P.U.F). Dans ce sens, on pourrait dire que l'enchevêtrement de la répétition dans la différence définit même la notion de structure. Voir : Lévi-Strauss, « Introduction », Anthropologie structurale. Paris, Plon, 1958, p. 19.

103 À propos de la critique autour du « centre de la structure », voir J. Derrida, « La structure, le signe et le jeu dans le discours des sciences humaines », dans L'Écriture et la différence. Paris, Seuil, 1967. Le « kantisme sans sujet transcendantal » c'est l'épigone forgé par Paul Ricœur sur Lévi-Strauss lors d'un entretien avec ce dernier : « Autour de La Pensée Sauvage. Repose à quelques questions », Esprit, $n^{\circ} 11,1963$

104 M. Foucault, « Débat sur le roman », op.cit., p. 371

105 Paul Imbs, L'emploi des temps verbaux en français moderne. Essai de grammaire descriptive, Paris, Klincksieck, 1960.

106 Oswald Ducrot ; Tzvetan Todorov, Dictionnaire Encyclopédique des sciences du langage, Paris, Éditions du Seuil, 1972, p. 390-391.
} 
grammairien). Si le temps porte sur l'énonciation, l'aspect porte sur le processus en quelque sorte « anonyme » du mouvement décrit.

Dans «Distance, aspect, origine », Foucault reprend cette discussion en sous-texte en identifiant ce temps indéterminé dans les ouvrages traités. À la liste des repères temporels flottants qu'il indique, on pourrait justement mettre en avant, comme noyau qui les rassemble, l'événement : « [chez Sollers, Baudry et Thibaudeau] la distinction des temps et des modes [...] ne renvoie que très indirectement à un calendrier; elle dessine des références, des index, des renvois où sont mises en jeu ces catégories de l'achèvement, de l'inachèvement, de la continuité, de l'itération, de l'imminence, de la proximité, de l'éloignement, que les grammairiens désignent comme catégories de l'aspect $\gg^{107}$. Soutenant le flux de ces mouvements, il n'y a pas un temps, mais un espace, qui est celui de la « distance ». Tout comme l'aspect complexifie le temps, la distance complexifie l'espace, et ces nouveaux axes remplacent les premiers : « la distance et l'aspect sont liés entre eux de façon plus serrée que l'espace et le temps ; [...] (l'aspect offrant, non le temps lui-même, mais le mouvement de sa venue ; la distance offrant non pas les choses en leur place, mais le mouvement qui les présente et les fait passer) $\gg^{108}$. De la sorte, l'origine ne peut qu'être cette plage continue de l'aspect, toujours en éloignement et en recul, étant elle-même un point vide qu'aucune genèse peut remplir.

Encore d'autres concepts seront forgés pour donner corps à cette nouvelle analyse littéraire foucaldienne. Le panorama déroulé à l'intérieur du langage amène à un remaniement de la définition de fiction, non dialectisée entre subjectif et objectif, qui se déploie dans la figure de l'intermédiaire (titre du livre de Sollers) et qui, insiste le philosophe, n'est pas « une région ou structure (mots trop engagés dans un mode de lecture qui ne convient plus) mais rapport constant, mobile, intérieur au langage lui-même $\gg^{109}$. L'intermédiaire, qu'ici conforme l'espace de la distance, est mentionné aussi dans «Débat sur le Roman », pour désigner l'accroc entre « penser et parler $\gg^{110}$. Ce sujet apparaît dans le cahier $n^{\circ} 5$ du Journal intellectuel noué à la discussion sur le partage entre pensée et philosophie ${ }^{111}$. Après les indications de Sade et de Hölderlin comme étant au seuil de la possibilité « d'une pensée libre par rapport à la philosophie », on lit : «Peut-être le Nouveau Roman n-a-t-il pas de philosophie mais se dirige vers une série d'expériences qui relèvent de la pensée $\gg^{112}$. Sans être ramenées à une psyché, les expériences (le rêve, la folie, la déraison, la répétition, le double, la déroute du temps, le retour, etc. ${ }^{113}$ ) ne nomment alors aucun phénomène en soi : le langage n'est pas ce qui dit ou traduit en mots une expérience, il est l'expérience ellemême - «pensée qui parle, parole pensante $»^{114}$. Cette conception de l'expérience, dissolvant autant l'objet que le sujet, s'oppose directement aux thèses essentialistes. Et elle est justement articulée par un « vocabulaire de la distance » : J'effacerai, pour laisser cette expérience à ce
qu'elle est (pour la traiter, donc, comme fiction,
puisqu'elle n'existe pas, c'est connu) tous les
mots contradictoires par quoi facilement on
pourrait la dialectiser: affrontement ou abolition
du subjectif et de l'objectif, de l'intérieur et de
l'extérieur, de la réalité et de l'imaginaire. Il
faudrait substituer à tout ce lexique du mélan-
ge le vocabulaire de la distance et laisser voir
alors que le fictif, c'est un éloignement propre
au langage [...] Il n'y a pas fiction parce que le
langage est à distance des choses; mais le
langage, c'est leur distance, la lumière où elles
sont et leur inaccessibilité [...] et tout langage
qui, au lieu d'oublier cette distance, se main-
tient en elle et la maintient en lui, tout langage
qui parle de cette distance en avançant en elle
est un langage de fiction ${ }^{115}$.

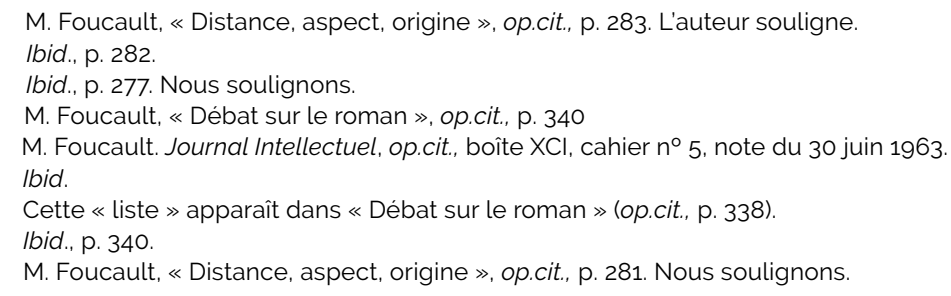


Ce sera de cette façon que, en parlant du « langage de l'expérience $»^{116}$, dans la note du 7 septembre 1963, Foucault affirmera encore : «On peut dire que les configurations du Nouveau Roman ont fait beaucoup pour la ramener au jour. Disparitions du sujet, dispersion du temps, répétition, double et dédoublement. Mais l'objet n'était sans doute que le point d'appui provisoire pour l'exercice et l'éclairage progressif de cela précisément : l'expérience $\gg^{117}$. L'enchevêtrement entre pensée et langage n'est jamais solidifié : la distance nomme exactement leur tension constante, elle est le mouvement de leur « partage » et de leur croisement. À la fin de l'article sur Tel Quel, le philosophe liera cette problématique spécifiquement au structuralisme, mais pour mieux réaffirmer son propre enjeu conceptuel :

Ce recul [absolu de l'origine] nous voue au partage [...] de la pensée et du langage ; en cette fourche [...] se dessine un espace où le structuralisme d'aujourd'hui pose [...] le regard de surface le plus méticuleux. Mais si [...] on lui demande d'où il nous vient, lui et [...] [ses] muettes métaphores, peut-être verrons-nous se dessiner des figures qui ne sont plus celles du simultané : les relations de l'aspect dans le jeu de la distance, la disparition de la subjectivité dans le recul de l'origine; ou, à l'inverse, ce retrait dispensant un langage déjà épars où l'aspect des choses brille à distance jusqu'à nous ${ }^{118}$.

La « métaphore » s'ajoute ainsi à la liste des opérateurs structuraux dont on a accompagné la critique : le centre vide de l'objet, la demeure insistante de la différence, la clôture du système et de la structure, l'emplacement des signes. Le ton de ces critiques, néanmoins, est encore très différent du type de rejet généralisant - et, surtout, stratégique - qu'on remarque chez le Foucault notamment à partir des années 1970.

Finalement, si le creux objectal a été remplacé par l'expérience, on assistera encore à d'autres déploiements pour la question de la « lacune » dans ce contexte. Regardons deux moments. Le premier se trouve dans le corpus publié et s'avère en connexion avec ce qui a été dit sur l'aspect dans son rapport avec le temps. Trois ans après « Distance, aspect, origine », Robbe-Grillet réapparaît sous la plume de Foucault dans le compte-rendu du livre Ouverture, de Thibaudeau, aussi de 1966. Dans ce texte, intitulé «À la recherche du présent perdu », Foucault remarque qu'entre la publication du livre de l'écrivain analysé précédemment (Une cérémonie royale) et le nouvel ouvrage « une certaine part de l'expérience littéraire a changé $\gg$. Et la portée de cette différence vient précisément d'une comparaison entre Une cérémonie royale et Robbe-Grillet, curieusement en les rapprochant sur la « lacune » de l'objet : « Un peu comme dans Le Voyeur ou La Jalousie, le récit s'organisait par fragments autour d'une plage laissée blanche: celle où résidaient jadis le "héros" ou l'événement" du récit classique $»^{119}$. Différemment, dans Ouverture, « le temps a basculé [...] Le présent, dans le roman de Thibaudeau, ce n'est pas ce qui ramasse le temps en un point pour offrir un passé restitué et scintillant; c'est, au contraire, ce qui ouvre le temps sur une irréparable dispersion $\gg^{120}$. Il en sera ainsi que le temps, qui dans le premier livre était une vibration de fragments débouchant sur une chronologie complètement vide, va devenir, dans le deuxième, une temporalité « présente », existante et repérable, mais « ruinée » ${ }^{121}$, discontinue. Il semble alors que ce changement littéraire, annoncé en 1966, portait justement sur le remplissement du vide d'avant.

Pour conclure, passons au deuxième moment, qui se trouve dans les manuscrits, où on verra ratifiée notre idée que, bien au-delà de ses critiques, c'est la philosophie foucaldienne même qui se construisait dans et par ses éloignements. Ainsi, il n'est peut-être pas surprenant que l'importance de Robbe-Grillet soit reprise dans le contexte déjà bien différent du Cours de Tunis, qui traite en grande partie du problème anthropologique dans les

\footnotetext{
${ }_{116}$ M. Foucault, Journal Intellectuel, op.cit., boîte XCl, cahier $n^{\circ} 5$, note du 7 septembre 1963

Ibid. Nous soulignons.

8 M. Foucault, «Distance, aspect, origine », op.cit., p. 284

Foucault, «À la recherche du présent perdu », L'Express, n 775, 25 avril - $1^{\circ}$ mai 1966, repris dans Dits et écrits, vol. 1, op.cit., pp. 504-

505, ici p. 504. Nous soulignons

120 Idem.

121 Ibid., p. 505
} 
sciences humaines. Dans ce Cours, on trouve une piste différente pour le schéma que nous venons d'esquisser, peut-être même à l'opposé de ce qu'il affirmait sur le « vide » chez l'écrivain en 1963 :

[Il s'agit d'une] littérature enfin où l'homme est à ce point absent, qu'elle peut se permettre de représenter cette absence

- Le Voyeur

- La Jalousie

- Et surtout Le Labyrinthe [...]

[Dans la littérature, la disparition de l'homme] n'est pas simplement un fait ; elle est désignée et représentée pour elle-même.

Toute l'œuvre de Robbe-Grillet peut être lue à partir de cette lacune qui crée dans le langage. dans les choses, dans le monde cette disparition de l'homme. Qu'est-ce que l'entrelacement des mots et des choses, si le démiurge humain lui fait défaut? [...]

Le romain de Robbe-Grillet est la présence manifeste de l'homme disparu'122.

Le vide excessif, « objectal », devient alors représentation exaltée, où cette littérature est la possibilité même pour la représentation de représenter sa propre fin. La lacune qui dérobait le mutisme de l'objet devient, en 1967, l'emplacement pour la célèbre absence de l'homme. Dans ce même sens, l'écrivain est mentionné aussi dans l'entretien «L'homme est-il mort? », de 1966, témoignant de l'entrecroisement entre pensée et savoir ${ }^{123}$. En tout cas, l'apparition de Robbe-Grillet dans un tel contexte demeure curieuse lorsqu'on se rappelle qu'une référence directe à l'analyse littéraire ou aux écrivains de Tel Quel était absente de la configuration contemporaine de la littérature décrite à la fin des Mots et les choses - là où Foucault thématisait justement le sol épistémique du formalisme contemporain.

\section{Considérations finales}

Nous avons présenté ici quelques éléments de la constellation théorique ouverte par l'analyse littéraire et par le structuralisme qui nous permettent une relecture d'une partie de la pro- duction foucaldienne des années 1960, mettant en question quelques vérités « anti-structurales » qui entourent son œuvre. À partir de nos fils conducteurs principaux - la distance, la structure et l'événement - nous avons souligné que le dialogue foucaldien avec le structuralisme se fait entre acceptation, refus, complexification et amélioration. La méthode d'analyse littéraire de Foucault entretient alors un profond contact avec ce qu'elle critique, puisqu'elle se construit complètement à l'instar de l'analyse structurale, en utilisant son mode d'opération pour forger, avec des altérations, ses propres concepts : c'est ce que nous avons voulu démontrer par les notions de réseau, d'isomorphisme, de mise en abîme, d'intermédiaire, entre autres, rassemblées autour du motif de la lacune chez Robbe-Grillet.

De ce fait, ce qui nous a intéressé ici était précisément la lecture de cette position (ou, en foucaldien, le repérage du sol de cette possibilité), et l'étude d'un tel débat avec le projet structural comme consistant en la distance même dans laquelle il a pu se poser et se réaliser. Si « l'infidélité » de lecture est aussi ce que nous apprend les écrits du philosophe, mettre en réseau Foucault et les écrits « structuralistes » d'autres auteurs, serait elle-même une procédure de désessentialisation de la critique de son œuvre. Et alors cette méthode de critique que nous avons voulu emprunter dans notre texte s'avère finalement très proche de la tâche de l'analyse littéraire défendue par le philosophe lui-même : « si la critique a un rôle [...] s'il peut être à la fois second et fondamental, c'est dans la mesure où il fait venir pour la première fois jusqu'aux mots ce réseau des œuvres qui est bien pour chacune d'elles son propre mutisme $\gg^{124}$.

Remerciements : Le développement de cette étude n'aurait pas été possible sans le contexte de travail du Groupe de Recherche sur les Archives Foucault (GRAF), dirigé par Orazio Irrera à l'Université Paris 8 Vincennes-Saint-Denis, que nous remercions vivement pour le soutien.

122 M. Foucault, Cours de Tunis, op.cit. Nous soulignons.

${ }_{123}$ M. Foucault, « L'homme est-il mort? », entretien avec C. Bonnefoy, Arts et Loisirs, n 38 , juin

1966, repris dans Dits et écrits, vol. 1, op.cit., pp. 540-544, ici p. 544.

124 M. Foucault, « Distance, aspect, origine », op.cit., p. 278. 


\section{Bibliographie}

BALIBAR, Étienne. Le structuralisme: une destitution du sujet? Revue de métaphysique et de morale, [S. l.], v. 1, n. 45. p. 5-22, 2005. https://doi.org/10.3917/rmm.051.0005

BARTHES, Roland. Michelet par lui-même, Paris, Éditions du Seuil, 1954.

BARTHES, Roland. Mythologies. Paris: Éditions du Seuil, 1957.

BARTHES, Roland. Sur Racine. Paris: Éditions du Seuil, 1963.

BARTHES, Roland. Essais critiques. Paris: Éditions du Seuil, 1964.

DELEUZE, Gilles. Différence et répétition. Paris: Presses Universitaires de France, 1968.

DELEUZE, Gilles. Logique du sens. Paris: Presses Universitaires de France, 1969.

DELEUZE, Gilles. À quoi reconnaît-on le structuralisme ? in L'île déserte et d'autres textes, Paris: Éditions de Minuit, 2002.

DERRIDA, Jacques. L'écriture et la différence. Paris: Éditions du Seuil, 1967.

DUCROT, Oswald; TODOROV, Tzvetan. Dictionnaire Encyclopédique des sciences du langage. Paris: Seuil, 1972.

FOREST, Philippe, Histoire de Tel Quel. 1960-1983. Paris: Éditions du Seuil, 1995

FOUCAULT, Michel. Histoire de la folie à l'âge classique. Paris: Gallimard, 1961.

FOUCAULT, Michel. Naissance de la Clinique. [S. l.]: P.U.F, 1963.

FOUCAULT, Michel. Les Mots et les choses. Une archéologie des sciences humaines, Paris, Gallimard, 1966.

FOUCAULT, Michel. L'Archéologie du savoir, Paris, Gallimard, 1969.

FOUCAULT, Michel. Dits et Écrits, 4 volumes, Paris, Gallimard, 1994.

FOUCAULT, Michel. La Grande étrangère. À propos de littérature, Paris, Éditions EHESS, 2013.

FOUCAULT, Michel. Folie, langage, littérature. Henri-Paul Fruchaud, Daniele Lorenzini et Judith Revel (éd.), Paris, Vrin, 2019.

FOUCAULT, Michel. Le Discours philosophique, Département de Manuscrits de la BnF, Fonds Foucault, NAF 28730, boîte LVIII.

FOUCAULT, Michel. Cours de Tunis, Département de Manuscrits de la BnF, Fonds Foucault, NAF 28730, boite LVIII. (M. Foucault, Le discours philosophique. Cours de Tunis 1966-1968, Orazio Irrera ; Daniele Lorenzini [éd.], Paris, Gallimard/Seuil/EHESS, collection. Hautes Études, [en préparation]).

FOUCAULT, Michel. Le Journal intellectuel, Département de Manuscrits de la BnF, Fonds Foucault, NAF 28730 , boite $\mathrm{XCl}$ et XCll.
FOUCAULT, Michel. Structuralisme et histoire, Département de Manuscrits de la BnF, Fonds Foucault, NAF 28730, boîte LXX.

GENETTE, Gérard. Sur Robbe-Grillet. Tel Quel, n. 8, 1962.

GIL, Marie. Foucault invente l'histoire littéraire. Fabula-LhT, n. zéro, 2005

GRANGER, Gilles-Gaston. Pensée formelle et sciences de l'homme. Paris: Aubier-Montaigne, 1967.

IMBS, Paul. L'emploi des temps verbaux en français moderne. Essai de grammaire descriptive. Paris: Klincksieck, 1960.

LE BLANC, Guillaume. La pensée Foucault. Paris: Ellipses, 2002.

LÉVI-STRAUSS, Claude. Anthropologie structurale. Paris: Plon, 1958.

RICOEUR, Paul. Autour de La Pensée Sauvage. Réponse à quelques questions, n. 11, Esprit, nov. 1963.

LORENZINI, Daniele. Foucault et la force des mots: de l'extralinguistique à la subjectivation. Phantasia, [S. l.], v. 8, 2019.

MANIGLIER, Patrice, «Introduction », in Patrice Maniglier (dir.), Le Moment philosophique des années 1960 en France, Paris, Presses Universitaires de France, 2011.

MANIGLIER, Patrice. Du mode d'existence des objets littéraires: enjeux philosophiques du formalisme, Les Temps Modernes, [S. l.], n. 676, p. 48-80, 2013. https:// doi.org/10.3917/ltm.676.0048

MANIGLIER, Patrice. La philosophie qui se fait. Dialogue avec Philippe Petit. Paris: Éditions du Cerf, 2019.

REVEL, Judith; G. BLANCO, Azucena. Michel Foucault: dire politique, dire littéraire, dire philosophique. Revue internationale de philosophie, dossier Michel Foucault: politique, littérature et philosophie, n. 292, 2/2020. https://doi.org/10.3917/rip.292.0007

RICARDOU, Jean. Pour une théorie du Nouveau Roman. Paris: Éditions du Seuil, 1971.

RICARDOU, Jean. Le Nouveau Roman. Paris: Seuil, 1973.

RICARDOU, Jean. Problèmes du Nouveau Roman. Paris: Éditions du Seuil, 1967.

ROBBE-GRILLET, Alain. Les Gommes. Paris: Éditions de Minuit, 1953.

ROBBE-GRILLET, Alain. Le Voyeur. Paris: Éditions de Minuit, 1955.

ROBBE-GRILLET, Alain. La Jalousie. Paris: Éditions de Minuit, 1957.

ROBBE-GRILLET, Alain. Pour un Nouveau Roman. Paris: Éditions de Minuit, 1963.

ROBBE-GRILLET, Alain. La littérature aujourd'hui. Tel Quel, Seuil, n. 14, 1963. 
ROBBE-GRILLET, Alain. Alain Robbe-Grillet au micro de Jean Thibaudeau, programme Entretiens avec chez France Culture, émission du 7 février 1975. Disponible sur: https://Www.franceculture.fr/litterature/ alain-robbe-grillet-sur-le-nouveau-roman. https://doi. org/10.2307/464689

SABOT, Philippe. La littérature aux confins du savoir. Sur quelques « dits et écrits » de Michel Foucault, in P.-F. Moreau (dir.). Lectures de Michel Foucault 3: Sur les Dits et écrits. Lyon: ENS Éditions, 2003. p. 17-33. (Coll. Theoria).

SABOT, Philippe. Notice (Raymond Roussel), in : M. Foucault, OEuvres, t. I, Gallimard, «Bibliothèque de la Pléiade », Paris, sous la direction de Gros F., avec Bert J.-F., Defert D., Delaporte Fr., Sabot Ph., Rueff M., Chevallier P., Harcourt B. et Senellart M., 2015. p. 1552-1662.

SABOT, Philippe. Dans les "Archives" de l'Archéologie. Relire Les Mots et les choses aujourd'hui. Rev. Filos. Aurora, Curitiba, v. 28, n. 45, p. 747-766, set./dez. 2016. https://doi.org/10.7213/1980-5934.28.045.DS01

SOLLERS, Philippe. A. Robbe-Grillet: Pour un Nouveau Roman. Tel Quel, [S. l.], n. 18, p. 93-94, 1964.

TEL QUEL, Déclaration. Tel Quel, [S. l.], n. 1, p. 3-4, 1960.

\section{Gabriela M. Jaquet}

Doctorante en philosophie/Université Paris-Est Créteil, chercheuse au GRAF/Université Paris 8 Vincennes-Saint-Denis.

\section{Norman R. Madarasz}

Professeur associé du programme de troisième cycle en philosophie et d'études de troisième cycle en lettres à la l'école des sciences humaines de PUCRS.

\section{Adresse postale}

Gabriela M. Jaquet

Avenue du Général de Gaulle, 61, 94000

Créteil, Paris, France

\section{Norman R. Madarasz}

Pontificia Universidade Católica do Rio Grande do Sul

Avenida Ipiranga 6.681, Prédio 8, $4^{\circ}$ andar.

Partenon, 90619-900

Porto Alegre, RS, Brasil 